The emission lines have an apparent breadth of $1.2 \mathrm{~A}$, indicating a considerable expansion velocity, or turbulence, in the "chromosphere" of the star. The breadth is so large that the measured position of the emission gives only an approximate velocity, which differs by $-8 \pm 4 \mathrm{~km} / \mathrm{sec}$ from that given by the absorption lines. In any case, the systematic motion of the chromosphere is small. The measured stellar velocity is $-26 \pm 1 \mathrm{~km} / \mathrm{sec}$, to be compared with $-26.9 \mathrm{~km} / \mathrm{sec}$, given in the Lick Catalogue of Radial Velocities. While variability of the stellar velocity has been suspected, ${ }^{2}$ it has apparently not been confirmed.

Our dispersion is too low to permit a theoretical investigation of the contour of the emission band or of the central reversal. The actual measured intensity of the emission is high. The apparent residual intensity, $R$, measured with respect to the interpolated stellar continuum, is of the order of 0.6 for the $K$ line. The instrumental contour has appreciably affected this value, and $R$ may be as high as 0.8 . In Arcturus, ${ }^{3}$ Wellmann finds $R=0.12$ for the $\mathrm{K}$ emission; the width, however, is approximately the same as in $56 \mathrm{Peg}$.

YeRKes OBSERVATORY

P. C. Keenan and J. L. Greenstein

June 1942

\title{
CONTINUOUS EMISSION IN THE SPECTRA OF GASEOUS NEBULAE*
}

\begin{abstract}
The visual continua in the spectra of three nebulae photographed with a narrow slit are not produced by the blending of atomic emission lines but must have a physical origin. Several strong $\mathrm{He}$ I emission lines just beyond the Balmer limit may tend to vitiate measurements of the intensity distribution in the Balmer continuum on plates taken with a wide slit.
\end{abstract}

In a recent study of the spectra of planetary nebulae Page ${ }^{1}$ used a very wide slit and was, therefore, unable to eliminate the possibility that the strong continuous spectrum in the visual region, $V_{c}$, is produced by an effect of blending of many faint emission lines. Because of the great importance of ascertaining whether the visual continuum, $V_{c}$, has a physical origin, we have obtained long exposures of three nebulae, using the quartz Cassegrain spectrograph with $f / 2$ camera and a very narrow slit. Each object was kept stationary on the slit, and in the case of the Orion nebula no stars brighter than visual magnitude 14 were on the slit.

Plate XXI shows spectrograms of the three nebulae obtained in January, 1942. In the first two (Orion nebula and IC 418) the general $V_{c}$ continuum is strong (of an intensity comparable to that of the Balmer continuum), whereas in IC 2165 it is very weak. Such differences between nebulae have been mentioned previously. These spectrograms prove conclusively that the visual continuum is not caused by the blending of many faint emission lines. Otherwise, the continuum observed with a narrow slit wrould show appreciable intensity fluctuations produced by the random distribution of the atomic lines. The emission is definitely continuous, and it appears likely that its

${ }^{2}$ Lick Obs. Bull., 6, 149, 1911.

${ }^{3}$ Zs. f. Ap., 19, 236, 1940.

* Contributions from the McDonald Observatory, University of Texas, No. 54.

${ }^{1}$ Ap. J., 96, 78, 1942. 